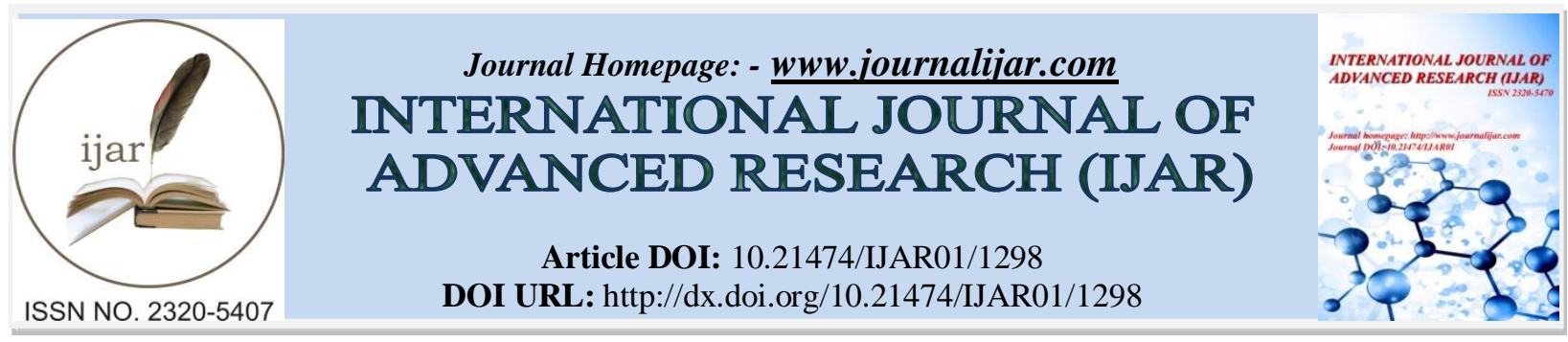

RESEARCH ARTICLE

\title{
INTERFERE OF NANOMATERIALS IN HUMAN LIFE.
}

Yogesh P Patil and Vishakha N Dhanwate.

Department of Engineering Sciences, AISSMS, Institute of Information Technology, Pune, MH India.

\section{Manuscript Info}

Manuscript History

Received: 15 June 2016

Final Accepted: 12 July 2016

Published: August 2016

Key words:-

Nanomaterials, Industrial and medical development, NP, pros and cons.

\section{Abstract}

Nanotechnology deals among the study of controlled formation, procedure and consumption of nanoparticles. As deliberation of several fields similar to energy, medical, environment, communication, cosmetics, agricultural, etc. there is requirement and scope for up gradation and improvements, nanotechnology can be a ray of hope for their progress. In the same way comfort level of human life style can be more superior in line with nature due to the advanced research and development in the field of nano materials. Because of distinctive constructive properties of materials in nano form than their bulk form, they are having incredible potential to transform the globe with technological progress, but same way we should responsive about the fact that nanoparticles may also have risky outcomes on human life. This paper presents pros and cones of nanomaterials in various fields with a case of CNT with reference to its medical and electronics applications.

Copy Right, IJAR, 2016,. All rights reserved.

\section{Introduction:-}

A variety of engineering materials are considered enormously for their properties in their nano form. Surface activity in nano form increases manifold than in bulk form due to increased surface area. The entire physics associated with material changes due to its transformation to the nano form [Limin Sun, et al, 2010]. In nanotechnology, material is introduced with billionth smaller dimensions in order to suit distinct applications of materials. These materials have impressive properties which are advantageous for technological development. As a result, the nano materials have become an integral part of individuals' life. Nanomaterials have wide range of appliacations, for example cosmetics [Alka Lohani et al, 2014] nano medicines [Anna Pratima Nikalje, 2015], nano conductors [Xinning Ho et al, 2013], catalysis [Beatriz Roldan Cuenya, 2010]. Not exactly the nanoparticles but also nanotubes, nanofibres may show carcinogenic pathology similar to asbestos [Grosse, et al, 2014]

\section{Pros and cones of Nanomaterials:-}

As nanotechnology is nothing but science of nano sized particles, they have various applications which are contemporary advancement as discussed elsewhere

\section{Applications of nano materials:-}

In their research Popov et al [Popov et al, 2005] and Patel et al [Anuradha Patel et al 2011] have discussed applications of nanoparticles for skin, nano sized titanium dioxide $\left(\mathrm{TiO}_{2}\right)$ and zinc oxides $(\mathrm{ZnO})$ are currently used 
in some sunscreens, as they absorb and reflect ultraviolet (UV) rays and yet are transparent to visible light. Carbon fibers are used in polymers to control or enhance conductivity [Allsopp et al, 2007] with applications such as antistatic packaging. For antistatic packaging clays are used Clays are containing the naturally occurring nanoparticles which are much more important in construction materials [Brezova et al, 2005]. These clay particles are based composites containing plastics and nano sized flakes of clay are also finding applications such as use in car bumpers. Coatings with thickness controlled at the nano scale like metal oxides for optoelectronic devices, or in catalytically active and chemically functionalized surfaces [Hidaka et al, 1997]. $\mathrm{TiO}_{2}$ functionalized as hydrophobic and antibacterial coating Cutting tools made of nanocrystalline materials, such as tungsten carbide, tantalum carbide and titanium carbide, are more wear and erosion resistant, and last longer than their conventional (large grained) counterparts. They are also finding applications in the drill used to bore holes in circuit boards [Abhilash et al, 2010]. Nanocrystalline zinc selenide, zinc sulphide, cadmium sulphide and lead telluride synthesized by sol gel techniques can be used for marketing in the form of displays. The huge market for large area, high brightness and flat panel displays, as used in television screens and computer monitors. With the growth in portable electronic equipment (mobile phones, navigation devices, laptop computers, remote sensors), there is great demand for lightweight, high-energy density batteries. In general, nanoparticles have a high surface area and hence provide higher catalytic activity. Nanoparticles used in textile industry [Y. H. Wong et al, 2006] Nanotechnology enables change in the degree of control in the production of nanoparticles and the support structure on which they reside. As preventing the corrosion of nanoparticles we get more life time of nanomaterials [Toshiyasu Nishimura, et al 2014]. It is possible to synthesize metal nanoparticles in solution in the presence of a surfactant to form highly ordered monodisperse films of the catalyst nanoparticles on a surface. This allows more uniformity in the size and chemical structure of the catalyst, which in turn leads to greater catalytic activity nano materials have exceptional mechanical properties, particularly high tensile strength and light weight. An obvious area of application would be in nanotube reinforced composites, with performance beyond current carbon fibre composites. Nanospheres of inorganic materials could be used as lubricants, in essence by acting as nano sized 'ball bearings'. It has been shown that magnets made of nanocrystalline yttrium samarium cobalt grains possess remarkable magnetic properties due to their extremely large grain interface area. This may lead to the development of applications in motors, analytical instruments like magnetic resonance imaging (MRI), used widely in hospitals, and microsensors. Nanocrystalline zirconium oxide (zirconia) is hard [J. M. Zieger et al, 1992]; wear resistant, bio corrosion resistant and bio compatible. It can be considered as better alternative to the existing biocompatible materials as they, sometimes, fail to continue their service throughout patient's life. Nanocrystalline ceramics, such as silicon nitride and silicon carbide, have been used in such automotive applications as high-strength springs, ball bearings and valve lifters, because they can be easily formed and machined, as well as exhibiting excellent chemical and high-temperature properties [Chang Huan et al, 2014].. Ceramics are hard, brittle and difficult to machine. However, with a reduction in grain size to the nanoscale, ceramic ductility can be increased. Zirconia, normally a hard, brittle ceramic, has even been rendered superplastic. Nano engineered membranes could potentially lead to more energy efficient water purification processes, notably in desalination by reverse osmosis. Nano filters can be developed to replace kidney functions. The new nanomedicine developed to address the need for better field treatments for massive human blood loss, which can cause cardiovascular collapse, also known as hemorrhagic shock. This potentially fatal condition is best treated with infusions of refrigerated blood and other fluids. Also the Ag NP is found anti cancer in addition to their antibacterial activity in cancer therapy [Kim D et al, 2012].

Lots of different formulations involving nanoparticles have been used for drug delivery purposes, including albumin, poly (D, L-lactic-co-glycolide) acid (PLGA), solid lipid formulations, cetyl alcohol/polysorbate nanoparticles [A. K Khan et al, 2014] gold nanoparticles [Amjadi M. et al 2014], polyalkylcyanoacrylate composites, magnetic iron oxide, methoxy poly (ethylene glycol)/poly ( $\varepsilon$-caprolactone) and gelatin. Albumin nanoparticles are already the subject of clinical studies for anticancer drug delivery purposes. Nanotechnology can augment agricultural production and boost food processing industry through applications of nanostructures [Angela G. King et al, 2010]. Nanosilver Ions and Particles are also used in antibacterial Activity [Georgios A et al, 2010]. Nanosensors are capable of detecting microbes, humidity and pollutants to very minute levels. Organic pesticides and industrial pollutants can be degraded into harmless and often useful components, through a process called photo catalysis using metal oxide semiconductor nanostructures. Leaching the molecules or atoms of metals come apart and leak into water or soil or the air. Leaching is when a less noble a less inert metal gets affected by moisture and the soil conditions. Inert metals like Ti are hardly attacked by acids except Nitric and HF. The respiratory tract system is the main route for dust entering the human body, followed by ingestion [Kanapilly G. M et al, 1980]. As the nano particles are inhaled, the probable sites for their deposition are brain, kidney, lungs [Barbara Rothen Rutishauser et al, 2007, Ludwig K et al 2007] pancreas and liver. They are likely to hamper the functions of affected 
organ by interfering with absorption ability and metabolic activities of the organ. With the small size and large surface area, nanoparticles can be an active group or exert intrinsic toxicity. However the absorption and deposition is always defined by the particle size in case of metal or inorganic nano particles [Hidaka, H, et al, 1997].

Nano-scale structures can involuntarily enter the gastrointestinal tract with food or when swallowing material cleared from the bronchi via mucociliary clearance. The oral intake of an average person is estimated to be $10^{12}$ to $10^{14}$ nano and microparticles daily [www.nanowork.com], with the overwhelming majority being silicates and titanium dioxide. In animal experiments, 50-100 nm-sized polystyrene particles pass through the gut wall and enter the lymphatic system and fullerenes, in contrast, tend not to be absorbed. Other studies, however, show no uptake into the vascular system via the gastrointestinal tract [Volkheimer G, 1974]. Materials with nano-size particles have always existed in nature and the atmosphere.

More recent uses of nanotechnology means that more and more man-made nanoparticles could in their life-time enter our atmosphere, soil or water environments. The risk of nanoparticles entering these environments, as well as the effects on human health from people having contact with the nanoparticles, needs to be assessed and researched in the following areas,

1. The detection of the particles in the environment.

2. The measurement of emissions of nano-particles.

3. The life-cycle of the particles in the environment.

4. The toxicity of the particles to the environment.

5. The impact on the immediate and longer range environment.

The main concern will be if any of the nanoparticles entering the environment are toxic or could become toxic to living species in the environment. For example, there is the possibility of nanoparticles being toxic to microorganisms in the soil and groundwater. Following on from this would be possible hazards from the nanoparticles or from consuming the microorganisms affected by the nanoparticles for fish, insects or mammals. There is also a risk to plants from nanoparticles which again could have a follow-on effect on the food chain. For example the deposition of atmospheric particles on crops could provide another route for toxic or reactive nanoparticles into the food chain.

Nanoparticles entering the environment may not initially be toxic to living species in the environment, but they could in their lifecycle become toxic. The nanoparticles could react to other substances in the environment, breakdown in the environment, provide a catalyst (speeding up) for the reactions already taking place, or prevent essential reactions taking place. For example, waste nanoparticles from a manufacturing plant entering a stream could alter the $\mathrm{pH}$ of the stream. Altering the $\mathrm{pH}$ of a stream can lead to metals that are not normally soluble dissolving, such as Aluminium. Aluminium in the water supply would in turn be toxic to living things in the stream [Tobias J. Brunner et al, 2006].

When considering the analysis of the nanoparticles consideration needs to be given to the size, shape, surface and bulk of the particles. These could all influence the properties of nanoparticles, so must all be considered when assessing the risk of the particles.

\section{Types of Nanoparticles:-}

The nanoparticles, natural or synthetic may occur in any of the following forms based on the conditions of their formation.

Liposomes:-

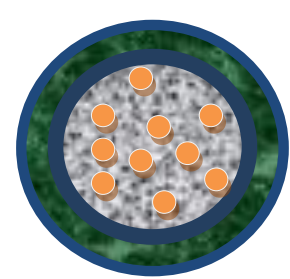

Figure 1:- Liposomes 
Liposomes are concentric vesicles which has two layers in which an aqueous volume is entirely enclosed by a membranous lipid which is composed of natural or synthetic phospholipids. Liposomes can be classified in terms of size, surface charge and number of bilayers [Abhilash M, 2010]

\section{S. L. Nanoparticles:-}

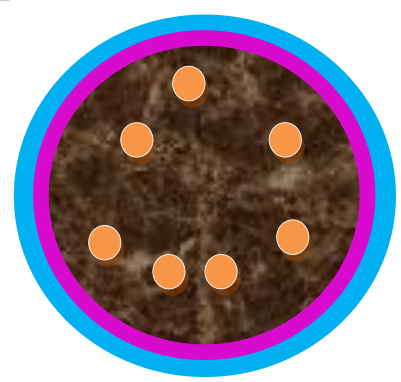

Figure 2:- S. L. Nanoparticles

Solid lipid nanoparticles (SLN) were developed as a substitute transporter or carrier system to emulsions, liposomes and polymeric nanoparticles as a colloidal carrier system for controlled drug delivery. Major motivation for their progress is the permutation of advantages from different carriers systems like liposomes and polmeric nanoparticles. SLN have been developed and investigated for parenteral, pulmonal and dermal application routes.

\section{Polymeric nanoparticles:-}

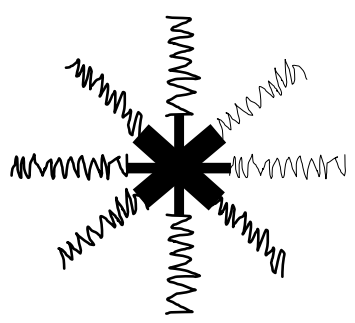

Figure 3:- Polymeric nanoparticles

In evaluation to SLN consists of a biodegradable polymer. Biocompatibility is an essential feature for potential application as tissue engineering, drug and gene delivery and new vaccination strategies. Most biodegradable polymers consists of synthetic polyesters like polycyanoacrylate or poly (D, L-lactide) and related polymers like poly (lactid acid) PLA or poly (lactide-co-glycolide) to give a few examples. Latest developments also include natural polymers like chitosan, gelatin, and sodium alginate to overcome some toxicological problems with the synthetic polymers.

\section{Dendrimers:-}

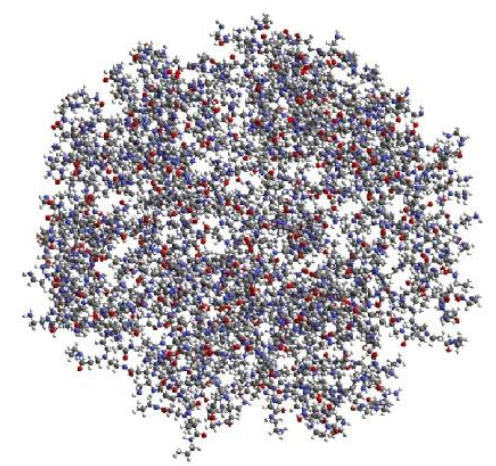

Figure 4:- Dendrimers 
Dendrimers is an exclusive category of polymers, which are highly branched with macromolecules whose size and shape can be precisely controlled. Dendrimers are fabricated from monomers using either convergent or divergent step growth polymerization. The well defined structure, monodispersity of size, surface fictionalization capability, and stability are properties of dendrimers that make them attractive carrier candidates [Abhilash M, 2010].

Nanocrystals:-

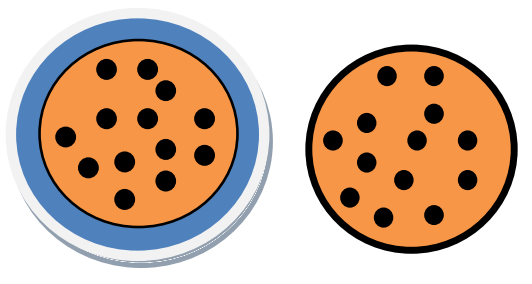

Figure 5:- Nanocrystals

Nanocrystals can be Nanosphere and nanocapsule which are aggregates of number of molecules that combine in a crystalline form, self-possessed of uncontaminated medicine with only a slim covering comprised of surfactant or combination of surfactants. Problems related to poorly soluble drugs like reduced bioavailability, improper absorption pattern may be set on through formulation as nanocrystals.

\section{Probability of entry to human body:-}

As existence of nano Particles is observed in different sizes, accordingly they affect on human life. Following are size classifications of nanoparticles,

Ultrafine $<100 \mathrm{~nm}$

Fine $>0.1 \mu \mathrm{m}$

Respirable (rat) $<3 \mu \mathrm{m}$

Respirable (human) $<5 \mu \mathrm{m}$

Inhalable (human) $<10-20 \mu \mathrm{m}$

\section{Detection Method:-}

There are several factors to be taken into account when aiming the detection and analysis. For example study of the gold nanoparticles in Zebrafish embryo model explained toxicity [Ganeshkumar $\mathrm{M}$ et al, 2012]. The influence of the environment on the particles and follow on effects must be considered alongside the toxicity of the nanoparticles themselves [Charles L. Geraci, et al, 2010, Chen Z, et al 2006]. There are various methods for the analysis of both physical and chemical properties of particles, however due to the fine size of the nanoparticles, all methods will not work properly or some methods require improvement to modify them. Both quantitative detection and analysis of the nanoparticles is required as experimental and quantum chemical approach is explained by pal et al. [Pal $\mathrm{R}$ et al, 2013].

Examples of methods that are successfully being used to measure nanoparticles in different environments include,

1) Scanning mobility particle sizer (SMPS).

2) Optical chromophore counting.

3) Resonant light scattering and Raman scattering techniques.

4) High Resolution Transmission Electron Microscopy (HRTEM).

5) Scanning Transmission Electron Microscopy (STEM).

\section{Case Study: Carbon Nano tube:-}

Carbon nanotubes are exclusive tubular structures of nanometer diameter and large length/diameter ratio. The nanotubes may consist of one up to tens and hundreds of concentric shells of carbons with adjacent shells separation of $0.34 \mathrm{~nm}$. Carbon nanotubes (CNTs) are flawless cylinders of one or more layers of graphene. Apart from the well-known graphite, carbon can build closed and open cages with honeycomb atomic arrangement. First such structure to be discovered was the C60 molecule by Kroto et al. [Kroto et al, 1985]. Researcher Iijima observed for the first time tubular carbon structures [Ijima et al, 1993]. CNT are unique because the bonding between the atoms is very strong and the tubes can have extreme aspect ratios. CNT are metallic or semiconducting, depending upon 
diameter and chirality. As a diameter increases, band gap increases. In the metallic state conductivity is very high. CNT is also good heat conductor. CNT are poor transmitter of electromagnetic energy. So their plastic composite can be used as light weight protector that is shielding material which protects the computers and electronic devices from weapons that emit electromagnetic pulse. Also because of low resistivity and high conductivity CNT used as interconnector, and due to their nano size, it is possible to obtain small electronic devices.

As we know hydrogen is one of the best sources for fuel cell, but due to its inflammable and explosive properties, there are lots of difficulties in hydrogen storage. But CNT is best alternative to store hydrogen in it. Many researchers and corporations have already developed CNT based air and water filtration devices. It has been reported that these filters can not only block the smallest particles but also kill most bacteria.

When human being carry forward the advances in technology, it is necessary to find out effects of products and byproducts on our day to day life. As our concern is with nanomaterials, therefore it is important to detect that how small size can affect the cells, whether barrier to brain is crossed by the particles and so on. On the other hand researcher believe that nanomaterial are useful for decreasing the environment pollution. But at the same time ongoing research at the various institutes reveal that CNT are as much lethal as asbestos may be [Sargent et al, 2014, Pritchard et al, 2003, Atsuya Takagi et al 2008, Negai et al 2011]. Though there is no any Mesothelioma patient is observed because of CNT till date, however researchers are reported that CNT induces Mesothelioma along with a positive control in mice [Atsuya Takagi et al 2008].

\section{Effect of Nano Particles on human health:-}

1. Nanoparticles may cause skin deceases [Adnan Nasir, 2010]

2. Nanoparticles may damage DNA [Gaetan Gogniat et al, 2007 Benedicte Trouiller et al, 2009,].

3. Nanoparticles may be transmitted through generations [Gaetan Gogniat et al, 2007].

4. Nanoparticles may deposit in lungs [Kreyling W. G, et al, 2002 Yong Chen et al, 2005].

5. Nanoparticles may be directly distributed to the brain via olfactory nerves [Oberdorster $\mathrm{G}$ et al, 2005]

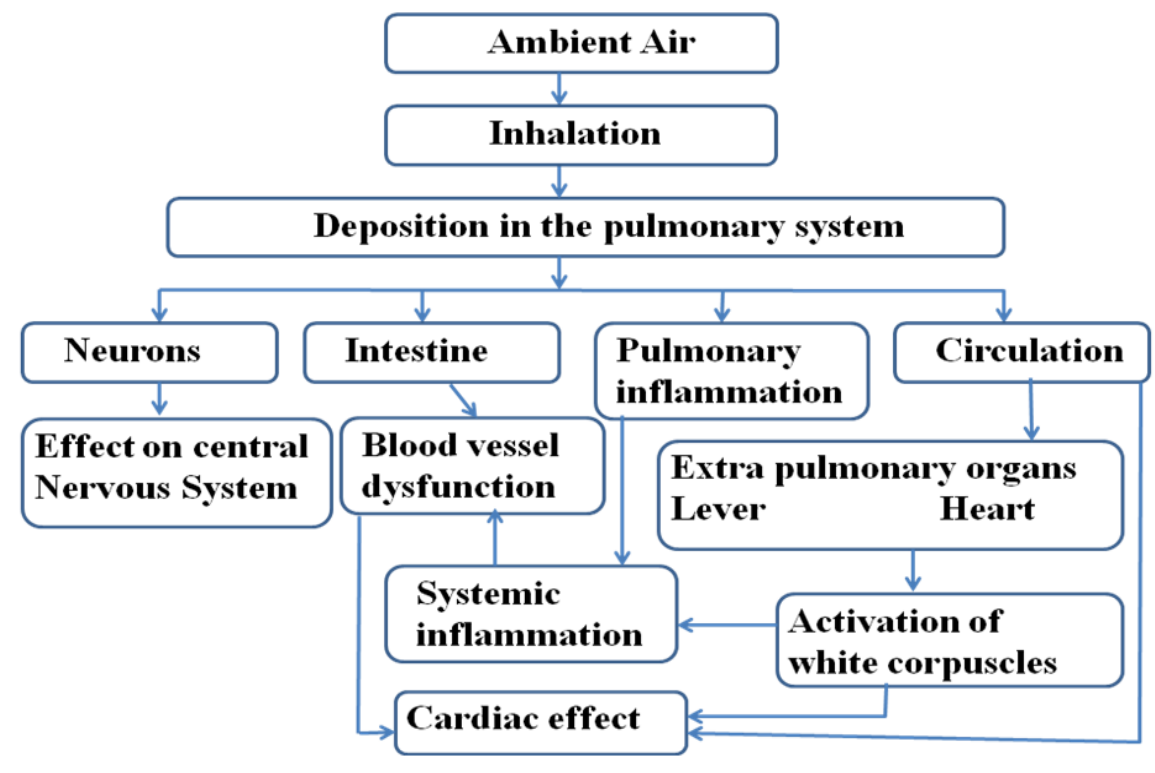

Figure 6:- Potential effects of inhaled ultrafine particles are according to Oberdorster G (Autonomous nervous system).Oberdorster [Oberdorster $\mathrm{G}$ et al, 2005] summarizes the effects on the body of inhaling nano-scaled ultrafine dusts. 
Clinical animal studies of ultrafine particles have shown pulmonary inflammation with histopathological change and translocation of particles to extra pulmonary tissues. Translocation of inhaled ultrafine particles in the bloodstream could affect endothelial function and promote thrombosis and other blood system problems, including increased blood coagulation. This phenomenon has been shown in hamsters but the situation in humans remains ambiguous. Such fine and ultrafine particles are responsible for Crohn's disease [Lomer M. C et al, 2002].

Epidemiological studies and volunteer studies of the human cardiovascular system have shown that the level of inhaled particles has direct effects on cardiovascular physiology, with alterations of cardiac rhythm and arterial diameter. Several epidemiological studies found a direct relationship between exposure to nano-scaled ultrafine dusts and respiratory and cardiovascular effects.

Significant relationships were established in several epidemiological studies showing that an increase in fine particle air pollution [S. Baruah et al, 2009], mainly due to vehicle emissions, led to an increase in morbidity and mortality of populations more fragile to respiratory [Nemmar A et al, 2003] and cardiac problems. Controlled clinical laboratory studies showed deposition of ultrafine dusts [Oberdorster $\mathrm{G}$ et al, 2005] throughout the pulmonary system [Nemmar A et al, 2002] accompanied by cardiovascular problems. Studies of coal miners exposed to ultrafine dusts showed accumulation of such dusts in the liver and spleen [Linglan Ma et al, 2006]. Accumulation was higher in miners exhibiting severe pulmonary problems, thus suggesting that damaged lungs or lungs with substantial deposits favors the passage of ultrafine particles to the blood system. Exposure of engineerwed nanoparticles to cells human lung shows adverse effect. [Ludwig K. Limbach et al, 2005].

Self defense mechanism against Nano Particles:-

The human body has various defense mechanisms to eliminate these undesirable foreign objects. Two processes are involved: chemical dissolution for soluble particles and physical translocation that is transport from one place to another, for insoluble particles or particles with low solubility. The mucociliary escalator eliminates the coarsest particles, which normally are deposited in the upper lungs, mainly in the tracheobronchial region. The tracheobronchial mucous membranes are covered with ciliated cells that form an escalator and expel the mucus containing the particles into the digestive system. Normally this is an efficient mechanism that eliminates particles from the respiratory tract in less than 24 hours, even ultrafine particles.

\section{Nano pollution control strategy:-}

1. Government should pay attention towards NP pollution and form some act so that no one can manufacture NP un-necessarily, as well as creating the waste while protecting the environment [S. Baruah et al, 2009].

2. Consumers should understand the 'nano' and then to decide their waste management technique so that nano waste could not impact our food chain.

3. Reuse, Reduce, and Recycle strategy [Angela G. King et al, 2010].

Nano pollutants are dangerous for all of us so we need to reduce on our reliability on nano related products and thus to check waste of nano. Also we should not manufacture nano related product just for business.

Reuse and recycle of nano a particle from fluid stream is possible using micro emulsion technique. However further research is highly needed because Nano tend to be highly active throughout their life span. Recovering and recycling nanoparticles is especially difficult because they tend to form complex, hard-to-separate mixtures with other substances.

Many researcher have also described the development of a special type of micro emulsion with mixture of oil and water (mayonnaise is an edible emulsion) that may solve this problem. In laboratory tests using cadmium and zinc nanoparticles, they showed how the oil and water in the micro emulsion separated into two layers when heated. One layer contained nanoparticles that could be recovered and the other contained none. The separation process is reversible and the recovered particles retain their shape and chemical properties, which is crucial for their reuse. Also in her report, Angela King [Angela G et al, 2010] reports the threat and opportunity behind the use of nano materials for biomedical applications and environmental protection through recycle reuse and reduce strategy.

\section{Conclusion:-}

Nanoscience refers to the world as it works on the atomic or molecular scale, from one to several hundred nanometers. Though there are some side effect due to nano particles are noted, nanoparticles are still associated with human growth. Which are connected with so many applications like small particle size means large surface area. 
Nanotube shaped catalysts may one day find application as industrial catalysts (speeds up certain chemical processes. In case of medical field, with the help of NP it is possible to repair selective membranes, blood substitutes, DNA modification etc. For industries, it is possible to create innovative plastics and ceramics with specialized and tunable properties based on structure. New scale of carbon based nanoelectronics will replace silicon based electronics. Nanotube wires are also used ballistic electron transport, as Electrons travel at $1 / 10 \mathrm{c}$ through wire with no resistance. However the controlled and discrete use of nano particles must be sought in coming years of development.

\section{Acknowledgement:-}

Authors are thankful to All India Shri Shivaji Memorial Society for the constant support through opportunities and the competitive work atmosphere provided.

\section{References:-}

1. A. K Khan, R Rashid, G Murtaza and A Zahra, Gold Nanoparticles: Synthesis and Applications in Drug Delivery, Tropical Journal of Pharmaceutical Research 13, 7 (2014) 1169-1177.

2. Abhilash M, Potential applications of Nanoparticles, International Journal of Pharma and Bio Sciences 1, 1 (2010) 1-12.

3. Adnan Nasir, Nanotechnology and dermatology, Part II- risks of nanotechnology, ELSEVIER, Clinics in Dermatology 28, (2010) 581-588.

4. Alka Lohani, Anurag Verma,1 Himanshi Joshi, Niti Yadav, and Neha Karki, Nanotechnology Based Cosmeceuticals, ISRN dermology 1 (2014) 1-14

5. Allsopp, M., Walters, A. \& Santillo, D, Nanotechnologies and nanomaterials in electrical and electronic goods: A review of uses and health concerns, GRL-TN-09 (2007) 1-22.

6. Amjadi M, Farzampour L. Fluorescence quenching of fluoroquinolones by gold nanoparticles with different sizes and its analytical application. J Luminesc, 145 (2014)263-268.

7. Angela G. King, Research Advances: Nanotechnology Research Attacks Cancer, Offers Big Development in Light Harvesting and Addresses the 3Rs: Recover, Recycle, and Reuse, Journal of Chemical Education 87, 9 (2010), 889-890.

8. Anna Pratima Nikalje Nanotechnology and its Applications in Medicine, medicinal Chemistry, 5, 2 (2015) 8189.

9. Anuradha Patel, Parixit Prajapati Rikisha Boghra, Overview on application of nanoparticles in cosmetics, Asian Journal of Pharmaceutical Sciences and Clinical Research, 1, 2 (2011) 40-55

10. Atsuya Takagi, Akihiko Hirose, Tetsuji Nishimura, Nobutaka Fukumori, Akio Ogata, Norio Ohashi, Satoshi Kitajima and Jun Kanno, Induction of mesothelioma in p53+/- mouse by intraperitoneal application of multiwall carbon nanotube The Journal of Toxicological Sciences 33, 1 (2008) 105-116.

11. Barbara Rothen Rutishauser, Robert N. Grass, Fabian Blank, Ludwig K. Limbach, Christian Mhlfeld, Christina Brandenberger, David O. Raemy, Peter Gehr, Wendelin J. Stark, Direct Combination of Nanoparticle Fabrication and Exposure to Lung Cell Cultures in a Closed Setup as a Method To Simulate Accidental Nanoparticle Exposure of Humans, Environmental Science \& Technology 43, 7, (2007) 2634-2640.

12. Beatriz Roldan Cuenya, Synthesis and catalytic properties of metal nanoparticles: Size, shape, support, composition, and oxidation state effects, Thin Solid Films 518 (2010) 3127-3150.

13. Benedicte Trouiller, Ramune Reliene, Aya Westbrook, Parrisa Solaimani, and Robert H. Schiestl, Titanium Dioxide Nanoparticles Induce DNA Damage and Genetic Instability In vivo in Mice Cancer Res, 69 (2009) 8784-8789.

14. Brezova, V., Gabcova, S., Dvoranova, D., Stasko, A. J Applications of nanoparticles, Journal of Photochem. and Photobiol, A Chemistry 79, (2005). 121-134

15. Chang Huan and Sun Shu-Qing, Silicon nanoparticles: Preparation, properties, and applications, Chin. Phys. B, 23, 8 (2014) 088102.1-14.

16. Charles L. Geraci, Vincent Castranova Challenges in assessing nanomaterial toxicology: a personal prospective, WIREs Nanomed Nanobiotechnol 2 (2010) 569-577.

17. Chen, Z.; Meng, H.; Xing, G.; Chen, C.; Zhao, Y.; Jia, G.; Wang, T.; Yuan, H.; Ye, C.; Zhao, F.; Chai, Z.; Zhu, C.; Fang, X.; Ma, B.; Wan, L Acute toxicological effects of copper nanoparticles in vivo Toxicology Letters, 163, 2(2006) 109-120. 
18. Gaëtan Gogniat and Sam Dukan, TiO2 Photocatalysis Causes DNA Damage via Fenton Reaction-Generated Hydroxyl Radicals during the Recovery Period Applied and Environmental Microbiology73, 23 (2007) 77407743

19. Ganeshkumar M, Sastry TP, Sathish Kumar M, Dinesh MG, Kannappan S, Suguna L. Sun light mediated synthesis of gold nanoparticles as carrier for 6- mercaptopurine: Preparation, characterization and toxicity studies in zebrafish embryo model. Mater Res Bull 47, (2012) 2113-2119.

20. Georgios A. Sotiriou and Sotiris E, Antibacterial Activity of Nanosilver Ions and Particles, Pratsinis Environmental Science \& Technology, 44, 14 (2010) 5649-5654

21. Grosse, Y., Loomis, D., Guyton, K.Z. Carcinogenicity of fluoro-edenite, silicon carbibe fibres and whiskers, and carbon nanotubes. Lancet Oncology, 15 (2014) 1427-28.

22. H.W. Kroto, J.R. Heath, S.C. O’Brien, R.F. Curl, R.E. Smalley, C60: buckminsterfullerene, Nature 318 (1985) 162-165

23. Hidaka, H., Horikoshi, S., Serpone, N., Knowland, Nanoparticles, I. J. of Photochem. and Photobiol. A: Chemistry 111, (1997) 205-213

24. http://www.oecd.org/dataoecd/37/19/37770473.pdf

25. http://www.physorg.com/news63466994.html

26. Ijima S, Ichihashi T. Single-shell carbon nanotubes of 1-nm diameter. Nature 363 (1993)603-605.

27. J. M. Zieger, G Sarcoid granulomatosis after many years of exposure to zirconium, "zirconium lung", Pathologe 13, 2 (1992) 104-109.

28. Kanapilly G. M. and Diel, J , Ultrafine $239 \mathrm{PuO}_{2}$ aerosol generation, characterization and short-term inhalation study in the rat, Health Phys 39, 3 (1980) 505-519.

29. Kim D, Jon S. Gold nanoparticles in image-guided cancer therapy. Inorgan Chim Acta, 393, (2012) 154-164.

30. Kreyling W. G, Semmler M, Erbe F, Mayer P, Takenaka S, Schultz H, Translocation of ultrafine insoluble iridium particles from lungs epithelium to extrapulmonary organs in size dependent but very low, J Tox Environ Health 65, 20 (2002) 1513-1530.

31. Lauterwasser (18 July 2007). OECD.org

32. Limin Sun, Laurence C. Chow, Stanislav A. Frukhtbeyn, Preparation and Properties of Nanoparticles of Calcium Phosphates with Various Ca/P Ratios, J. Res. Natl. Inst. Stand. Technol, 115, 4 (2010) 243-255.

33. Linglan Ma, Jinfang Zhao, Jue Wang, Jie Liu, Yanmei Duan, Huiting Liu, Na Li, Jingying Yan, Jie Ruan and Han Wang, The Acute Liver Injury in Mice Caused by Nano-Anatase TiO2, Nanoscale Research Letters 4, 11 (2006) 1275-1285

34. Lomer M. C, Thompson, R. P. and Powell, J. J, Fine and ultrafine particles of the diet: influence on the mucosal immune response and association with Crohn's disease, Proc Nutr Soc 61, 1 (2002) 123-130.

35. Ludwig K. Limbach, Peter Wick, Pius Manser, Robert N. Grass, Arie Bruinink, and, Wendelin J. Stark, Exposure of Engineered Nanoparticles to Human Lung Epithelial Cells: Influence of Chemical Composition and Catalytic Activity on Oxidative Stress Environmental Science \& Technology 41,11, (2007) 4158-4163.

36. Ludwig K. Limbach,, Yuchun Li, Robert N. Grass,, Tobias J. Brunner,, Marcel A. Hintermann,, Martin Muller,, Detlef Gunther, and, Wendelin J. Stark' Oxide Nanoparticle Uptake in Human Lung Fibroblasts: Effects of Particle Size, Agglomeration, and Diffusion at Low Concentrations, Environmental Science \& Technology 39, 23(2005) 9370-9376.

37. Nagai, H., Okazaki, Y., Chew, S.H., et al. Diameter and rigidity of multiwalled carbon nanotubes are critical factors in mesothelial injury and carcinogenesis. Proceedings of the National Academy of Sciences USA, 108, E (2011) 1330- 1338

38. Nemmar A, Hoylaerts MF, Hoet PHM, Dindsdale D, Smith T, Xu H, Ultrafine particles affect experimental thrombosis in an in vivo hamster model. Am J Respir Crit Care Med 166 (2002) 998-1004.

39. Nemmar A, Hoylaerts MF, Hoet PHM, Vermylen J, Nemery B, Size effect of intratracheally instilled particles on pulmonary inflammation and vascular thrombosis, Toxicol Appl Pharm 186 (2003) 38-45.

40. Nishimura, T. Electrochemical behavior and structure of rust formed on $\mathrm{Si}$ and $\mathrm{Al}$ bearing steel after atmospheric exposure. Corr. Sci. 52 (2010) 3609-3614.

41. Oberdorster G, Maynard A, Donaldson K, Castranova V, Fitzpatrick J, Ausman K, Principles for characterizing the potential human health effects from exposure to nanomaterials: elements of a screening strategy. Part Fibre Toxicol 2, 1 (2005) 1-60.

42. Oberdorster G, Oberdorster E, Oberdorster J, Nanotoxicology: an emerging discipline evolving from studies of ultrafine particles. Environ Health Perspect 113 (2005) 823-839.

43. P. V. AshaRani, Grace Low Kah Mun, Manoor Prakash Hande and Suresh Valiyaveettil, Cytotoxicity and Genotoxicity of Silver Nanoparticles in Human Cells , ACS Nano,3, 2 (2009) 279-290. 
44. Pal R, Panigrahi S, Bhattacharyya D, Chakraborti AS. Characterization of citrate capped gold nanoparticlequercetin complex: Experimental and quantum chemical approach. J Mol Struct, 1046 (2013) 153163.

45. Popov AP, Lademann J, Priezzhev AV, Myllyla R, “ Effect of size of TiO2 nanoparticles embedded into stratum corneum on ultraviolet- A and ultraviolet-B sun-blocking properties of the skin" J Biomed Optics, 10 (2005) 1-9.

46. Pritchard, J.B, French, J.E., Davis, B.J., et al. The role of transgenic mouse models in carcinogen identification. Envrionmental Health Perspectives, 111, (2003) 444-454.

47. Ron Gill, Itamar Willner, Itzhak Shweky, and Uri Banin, Fluorescence Resonance Energy Transfer in CdSe/ZnS-DNA Conjugates: Probing Hybridization and DNA Cleavage J. Phys. Chem. B, 109, 49 (2005) 23715-23719.

48. S. Baruah and J. Dutta Nanotechnology applications in Sensing and Pollution Degradation in Agriculture Environmental Chemistry Letters 7 (2009) 191-205

49. Sargent, L.M., Porter, D.W., Staska, L.M., et al. Promotion of lung adenocarcinoma following inhalation exposure to multi-walled carbon nanotubes. Particle and Fibre Toxicology, (2014), 11-13.

50. Source: http://www.nanowork.com/nanotechnology/reports/reportpdf/report61.pdf

51. Tobias J. Brunner, Peter Wick, Pius Manser, Philipp Spohn, Robert N. Grass, Ludwig K. Limbach, Arie Bruinink, and, Wendelin J. Stark, In Vitro Cytotoxicity of Oxide Nanoparticles, Comparison to Asbestos, Silica, and the Effect of Particle Solubility, Environmental Science \& Technology 40, 14 (2006) 4374-4381.

52. Toshiyasu Nishimura, Vedarajan Raman, Corrosion Prevention of Aluminum Nanoparticles by a Polyurethane Coating, Materials 7 (2014),4710-4722.

53. Volkheimer G, Passage of particles through the wall of the gastrointestinal tract, Environ Health Perspect 9 (1974) 215-225.

54. Warheit, D. B. Nanoparticles: Health impacts, Materials today, 7, 2 (2004) 32-35.

55. Xinning Ho and Jun Wei, Films of Carbon Nanomaterials for Transparent Conductors, Materials 6 (2013) 2155-2181.

56. Y. H. Wong, C. W. M. Yuen, M. Y. S. Leung, S. K. A. K , and H. L. I. Lam, Selected applications of nanotechnology in textiles AUTEX Research Journal 6, 1 (2006) 1-8.

57. Yong Chen, Lisong Yang, Chao Feng and Long-Ping Wen, Nano neodymium oxide induces massive vacuolization and autophagic cell death in non-small cell lung cancer NCI-H460 cell Biochemical and Biophysical Research Communications 337, 1 (2005) 52-60. 